\title{
On the Performance of Bed-Integrated Ballistocardiography in Long-Term Heart Rate Monitoring of Vascular Patients
}

\author{
Christoph Hoog Antink ${ }^{1}$, Yen Mai ${ }^{2}$, Jukka Ranta ${ }^{3}$, Adrian Tarniceriu ${ }^{4}$, Christoph Brüser ${ }^{1}$, \\ Steffen Leonhardt ${ }^{1}$, Niku Oksala ${ }^{2,5}$, Antti Vehkaoja ${ }^{2}$ \\ ${ }^{1}$ Medical Information Technology, Helmholtz-Institute for Biomedical Engineering, \\ RWTH Aachen University, Aachen, Germany \\ ${ }^{2}$ Faculty of Medicine and Health Technology, Tampere University, Finland \\ ${ }^{3}$ Emfit Oy, Vaajakoski, Finland, ${ }^{4}$ PulseOn SA, Neuchatel, Switzerland \\ ${ }^{5}$ Vascular and Interventional Radiology Centre, Tampere University Hospital, Tampere, Finland
}

\begin{abstract}
The application of ballistocardiography (BCG) for unobtrusive monitor of (beat-to-beat) heart rate in sleeping subjects has seen increased attention in recent years. While most studies are performed on healthy volunteers, some studies have included subjects with sleep-related problems. Even so, little attention has been payed to patients suffering from cardiovascular diseases. In addition, most studies are either limited to short laboratory measurements or overnight recordings of sleeping subjects.

In this work, we present preliminary results on beatto-beat interval estimation of long-term ( $>21 \mathrm{~h})$ BCG recordings in a hospital environment. Measurements were obtained from five subjects after peripheral vascular surgery or endovascular interventions and compared to Holter recordings. The commercially available sensor Emfit QS as well an augmented version of the CLIE interval estimation algorithm were used. Using the proposed approach, an average relative beat-to-beat interval error of $3.8 \%$ at a coverage of $61.5 \%$ was achieved. Error and coverage were found to vary from subject to subject as well as time of day. On average, findings are comparable though inferior to results reported on insomniac subjects in a sleep laboratory environment.
\end{abstract}

\section{Introduction}

Unobtrusive night-time monitoring with bed-integrated ballistocardiographic (BCG) sensors has gained interest in wellness domain as it enables easy-to-use self-monitoring of sleep. So far, the use of BCG monitoring in clinical applications has been limited. A potential clinical application is unobtrusive monitoring of patients who are staying in beds in the regular hospital ward.

Currently, monitoring of such patients is relatively sparse or unsystematic, and is mainly performed visually or with intermittent measurements. Continuous monitoring of hospital patients would be beneficial in the detection of deterioration of a patient's condition, which, if achieved early, could enable preventive measures.

The BCG technique has been proven adequately accurate for heart rate variability measurement with healthy subjects and bed-integrated [1-3] as well as wearable seismocardiography (SCG) sensors [4]. Brüser et al. achieved $0.61 \%$ relative error $\bar{E}_{\text {rel }}$ with $85 \%$ coverage in beat-tobeat interval (BBI) estimation with eight healthy subjects. Corresponding numbers in the study by Kortelainen et al. were $0.4 \%$ and $88 \%$ with six subjects. The same studies also included groups of patients suffering from insomnia or other sleep related disorders. In these groups, the performance of BCG monitoring was decreased to $1.8 \%$ relative error and $80 \%$ coverage with 28 subjects [2] and $1.61 \%$ relative error and $69 \%$ coverage with 25 subjects [1]. For a detailed review of BCG and SCG applications as well as differences between the methods, we refer to [5].

In high-risk cardiovascular patients, the technology may face further reduction in accuracy, as the BCG signal complex induced by a heartbeat is often weaker and less consistent in shape. Kaisti et al. [6] reported over ten-fold increase in RMS error from $5.6 \mathrm{~ms}$ to $60.3 \mathrm{~ms}$ in study groups consisting of 29 healthy subjects and 12 coronary artery disease patients with a wearable seismocardiographic measurement method using a 6 degree of freedom motion measurement unit. The BBI signal coverage was $93.4 \%$ and $92.6 \%$ for the two groups, respectively.

In this work, we studied the performance of BBI estimation from BCG signals recorded with the commercially available Emfit QS monitoring system (Emfit Oy, Finland) in five patients who underwent endovascular interventions or open vascular surgical procedures. Typically, these patients have several comorbidities (such as diabetes, hyper- 
tension, dyslipidemia, coronary artery disease, cerebrovascular disease) and may have reduced cardiac function. For all patients, long-term recordings $(>21 \mathrm{~h})$ including dayand nighttime were acquired. For BBI estimation, the previously developed CLIE algorithm [1] was used and augmented with an iterative estimation approach.

\section{Data}

The data of five post-operative vascular patients was selected for the analysis. The patient recordings were made in the vascular surgery ward of the Tampere University Hospital. The subjects were monitored for approximately 24 hours with an EMFit QS bed sensor. A five-lead Holter monitor, Faros 360 manufactured by Bittium Biosignals, was used to record the reference ECG signal. The bed sensor was placed between the hospital bed mattress and the bed frame. The sampling rate of the BCG signal obtained with the Emfit QS system was $100 \mathrm{~Hz}$ and the signal was bandlimited from 1 to $5 \mathrm{~Hz}$. Ambu Blue sensor L-00-S electrodes were used for the ECG recording. A sampling rate of $1 \mathrm{kHz}$ was used with ECG.

The average age of the subjects was 65.0 years. Four of the subjects were male. The subjects had undergone different vascular and endovascular procedures: one lower limb endografting, one abdominal aortic endografting, two carotid endarterectomies, and one femoropopliteal bypass surgery. A favorable statement was obtained from the Regional Ethical Committee of Pirkanmaa Hospital District (R17027) for the study.

\section{Algorithm}

The Continuous Local Interval Estimator approach used in this paper is described in detail in [1]. In short, self-similarity of consecutive heartbeats is assessed using lag-adaptive versions of the short-term autocorrelation $S_{\text {LASTA }}[i, \eta]$, the maximum amplitude pairs $S_{\mathrm{MAP}}[i, \eta]$ and the average magnitude difference function $S_{\mathrm{AMDF}}[i, \eta]$. For each window $i$ of the BCG-signal, all functions give a probability that a candidate shift $\eta$ of the signal corresponds to the actual BBI, $\eta_{\mathrm{opt}, i}$. All three functions are fused using a Bayesian approach,

$$
\begin{aligned}
S_{\mathrm{f}}[i, \eta] & =S_{\mathrm{LASTA}}[i, \eta] \cdot S_{\mathrm{MAP}}[i, \eta] \cdot S_{\mathrm{AMDF}}[i, \eta], \\
\eta_{\mathrm{opt}, i} & =\underset{\eta}{\arg \max }\left[S_{\mathrm{f}}[i, \eta]\right] .
\end{aligned}
$$

Thus, the CLIE algorithm makes no prior assumptions about BBIs and was proven to be capable of processing even severely arrhythmic data [7]. The only tunable parameter of the original CLIE is the threshold of selfsimilarity, $q_{\mathrm{th}}$. Only candidate intervals with a enough self-similarity $\left(S_{\mathrm{f}}\left[\eta_{\mathrm{opt}, i}\right] / \sum_{\eta} S_{\mathrm{f}}[\eta]>q_{\mathrm{th}}\right)$ are accepted as valid intervals, increasing accuracy while reducing temporal coverage. However, in the present full-day data of partially awake, non-healthy subjects, significant outliers were found and the algorithm was modified to increase robustness at the cost of generality.

To remove outliers in the beat-to-beat interval estimates, a straight-forward thresholding algorithm was applied. If beats exhibited a relative deviation from the median estimation bigger than $\Delta_{\max }$, the detected interval was considered an outlier and removed from analysis.

As show in previous work, the inclusion of an adaptive prior could improve beat-to-beat estimation in low-quality signals [8]. Thus, a similar approach was applied. In this work, an iterative estimation approach was developed: In the first pass, CLIE was used to estimated beat-to-beat intervals without prior assumption about the heart rate. Next, the detected intervals $\eta_{\mathrm{opt}, i}$ were median filtered with a filter of width $n_{\text {median }}$,

$$
\hat{\eta}_{\mathrm{opt}, i}=\operatorname{medianfilter}\left(\eta_{\mathrm{opt}, i}, n_{\text {median }}\right)
$$

In the second pass, an adaptive prior was used in the interval estimation,

$$
\begin{aligned}
S_{\text {prior }}\left[\eta, k, \hat{\eta}_{\mathrm{opt}, i}\right] & =\frac{1}{2 k} e^{-\frac{\left|\eta-\hat{\eta}_{\mathrm{opt}, i}\right|}{k}}, \\
S_{\mathrm{f}}^{*}\left[i, \eta, k, \hat{\eta}_{\mathrm{opt}, i}\right] & =S_{\mathrm{f}}[i, \eta] \cdot S_{\mathrm{prior}}\left[\eta, k, \hat{\eta}_{\mathrm{opt}, i}\right] .
\end{aligned}
$$

Based on previous observations [9], a Laplacian distribution with variable scale $k$ was chosen.

\section{Results and Discussion}

Without post processing, a mean absolute estimation error over all patients of $46.36 \mathrm{~ms}$ at a temporal coverage of $47.87 \%$ was observed for a fixed quality threshold $q_{\mathrm{th}}=0.4$ as used in previous studies. Thus, iterative estimation and outlier detection as described above were applied. The concept is visualized for an excerpt of one recording in Figure 1.

As can be seen from Figure 1, using the adaptive prior significantly reduces outliers so that only few intervals are excluded in the thresholding process. Nevertheless, detailed analysis shows that some ground truth intervals, presumably from areas of non-sinus rhythm are excluded in the thresholding process.

Figure 2 presents an analysis of the algorithms sensitivity towards a variation of the outlier threshold $\Delta_{\max }$ and the scale of the Laplacian prior $k$ for a fixed quality threshold. As expected, decreasing $\Delta_{\max }$ decreases both coverage and mean absolute error. A similar behaviour is observed for $k$ : The smaller $k$, the higher both error and coverage. This can be explained by the fact that a small scale of the prior distribution leads the algorithm, given a fixed $q_{\mathrm{th}}$, to be more confident about any detected interval. 

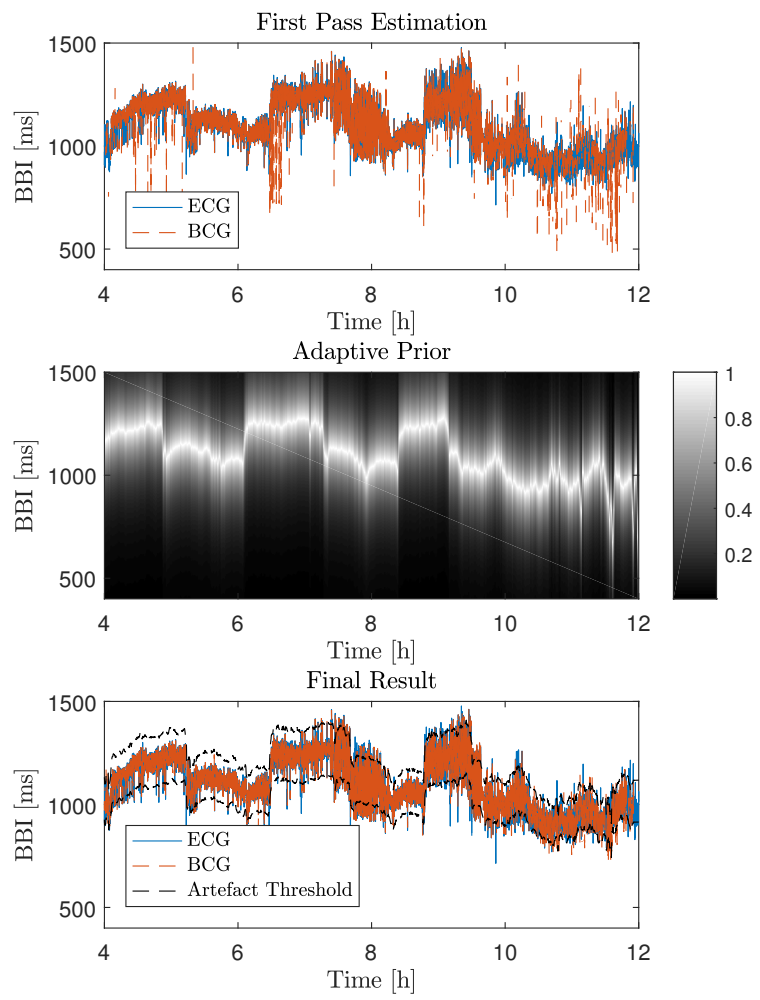

Figure 1. The effect of the proposed adaptive prior method on BBI estimation. Top: Estimated Intervals from BCG vs. ground truth intervals from Holter ECG. Middle: Resulting adaptive Prior. Bottom: Resulting interval estimation and artefact threshold for $\Delta_{\max }=10 \%$.

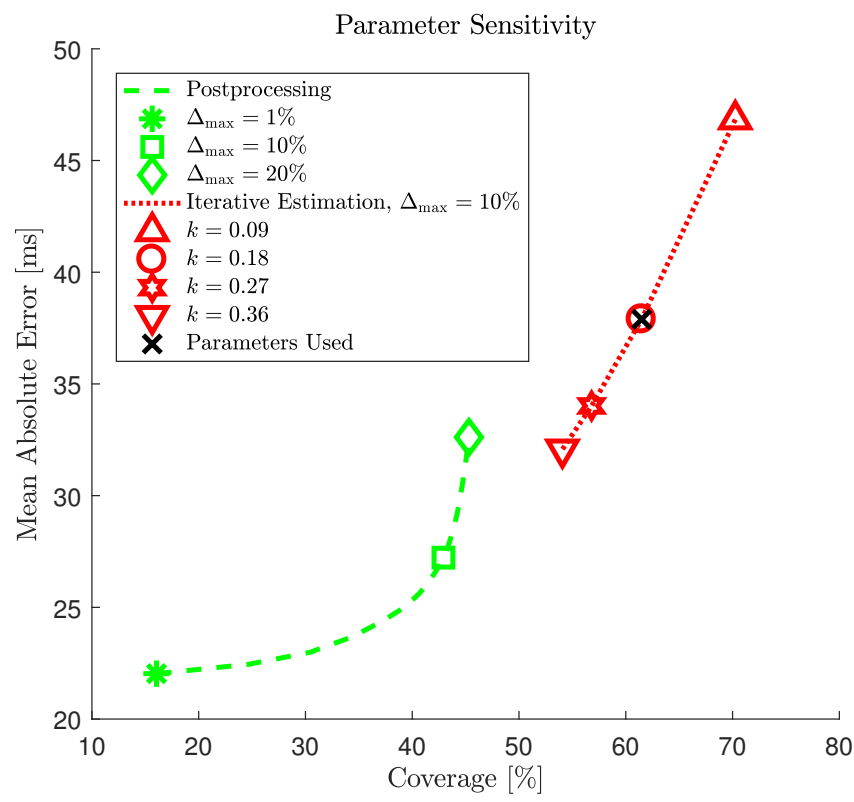

Figure 2. Sensitivity analysis of the algorithm on the outlier threshold $\Delta_{\max }$ and the scale of the Laplacian prior $k$.
The number of beats used to calculate the median interval $n_{\text {median }}$ was varied from 51 to 301 and was found to have little influence on coverage and error.

The patient data was processed using the parameter set marked in Figure $2\left(\Delta_{\max }=10 \%, k=0.18\right)$. Table 1 shows the results for each subject in terms of coverage and error as well as the usable duration, i.e. the period where both Holter ECG and bed sensor BCG was available. The coverage is a percentage value of this duration value.

\begin{tabular}{ccccc}
$\begin{array}{c}\text { Patient } \\
\text { ID }\end{array}$ & $\begin{array}{c}\text { Duration } \\
{[\mathrm{h}]}\end{array}$ & $\begin{array}{c}\text { Coverage } \\
{[\%]}\end{array}$ & $\begin{array}{c}\bar{E}_{\text {abs }} \\
{[\mathrm{ms}]}\end{array}$ & $\begin{array}{c}\bar{E}_{\text {rel }} \\
{[\%]}\end{array}$ \\
\hline 1 & 21.66 & 66.48 & 40.42 & 4.1 \\
2 & 21.57 & 58.03 & 38.84 & 3.8 \\
3 & 21.25 & 48.85 & 44.68 & 4.4 \\
4 & 22.77 & 61.80 & 41.12 & 4.1 \\
5 & 22.35 & 72.26 & 24.41 & 2.4 \\
\hline Mean & 21.92 & 61.48 & 37.89 & 3.8 \\
Gross Average & & 61.35 & 37.46 & 3.7
\end{tabular}

Table 1. Duration, coverage, mean absolute error $\bar{E}_{\text {abs }}$ as well as relative error $\bar{E}_{\text {rel }}$ of BBI estimation.

As can be seen in Table 1, the results are quite variable. For example, patient ID 3 exhibits the worst results both in terms of coverage $(48.85 \%)$ and error $(4.4 \%)$. On the other hand, patient ID 5 shows the best results, both in terms of coverage $(72.26 \%)$ and error $(2.4 \%)$. While the coverage of the best subject (ID 5) is comparable to previous data of sleeping subjects, the reported relative error is about three times higher than those of normal sleepers but comparable to insomniac patients. On average, however, the error has increased by a factor of approximately two to three compared to results from insomniacs without known cardiac problems.

In Figure 3, the error in terms of the time of day is shown for subject 2 . For better visualization, the median absolute error in a window of 301 beats (approximately 5 minutes) is presented. As can be seen, the error varies dramatically depending on the time of day. The error is comparatively low at the beginning of the measurement at $7 \mathrm{pm}$. At midnight, the error further drops as the subject presumably falls asleep. Between 4 am and 5 am, spikes in the error are visible, and the error starts increasing from then on. Starting at 8 in the morning, more spikes in the estimation error start to appear.

\section{Conclusion and Outlook}

While the presented results show that continuous monitoring of high-risk cardiovascular, non-sleeping subjects with BCG is possible, several challenges still need to be overcome. Similar to findings by other groups [6], the results obtained from our subjects are inferior to those ob- 

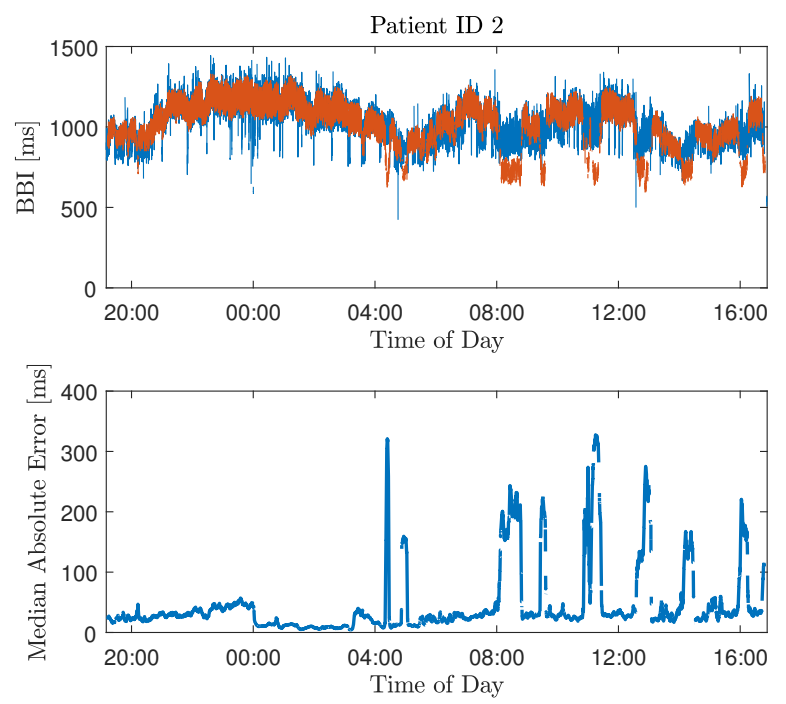

Figure 3. Median absolute error in a window of 301 beats for patient ID 2 over the time of day.

tained in sleep laboratory studies $[1,10]$, although a similar sensor and the same algorithm is used. Further development made to the algorithm was able to compensate the performance decrease partially. For one, the diseases themselves might have influences on the signal quality, thus leading to inferior results. For another, awake subjects will probably exhibit far more motion artifacts than sleeping ones. Similar observations were made comparing healthy controls to insomniac patients in the sleep laboratory [1]. In addition, patients may be in pain, delusional, they may be restless, or some medical procedures may be done to them while they are in the bed. Finally, during daytime, motions might be very subtle and even periodic, for example when subjects are talking. Thus, these artifacts may not be detected easily by thresholds on signal amplitude and self similarity measures. Future work should focus on distinguishing whether the signal quality or the state of wakefulness (as Figure 3 suggests) has the most significant influence on the estimation quality. Moreover, a larger cohort of subjects needs to be analyzed. In the current study, the nominal sampling rate of the devices was used. However, visual inspection revealed varying misalignment of BBI estimation in certain parts of the measurements. This hints at non-constant drifts in sampling time as observerd by other groups [11], which needs to be accounted for in future works. Finally, as subtle motion artifacts are a likely cause of a decreased performance, novel algorithmic concepts need to be developed to detect those.

\section{Acknowledgements}

The authors gratefully acknowledge financial support provided by the German Research Foundation
[Deutsche Forschungsgemeinschaft (DFG), LE 817/26-1] and Academy of Finland (grant numbers 310617, 310618, and 292477.)

\section{References}

[1] Brüser C, Winter S, Leonhardt S. Robust Inter-Beat Interval Estimation in Cardiac Vibration Signals. Physiological Measurement 2013;34(2):123-138.

[2] Kortelainen J, Gils MV, Pärkkä J. Multichannel Bed Pressure Sensor for Sleep Monitoring. In Computing in Cardiology, volume 39. 2012; 313-316.

[3] Vehkaoja A, Rajala S, Kumpulainen P, Lekkala J. Correlation Approach for the Detection of the Heartbeat Intervals using Force Sensors Placed Under the Bed Posts. Journal of Medical Engineering and Technology 2013;37(5):327-333.

[4] Di Rienzo M, Vaini E, Lombardi P. An Algorithm for the Beat-to-Beat Assessment of Cardiac Mechanics during Sleep on Earth and in Microgravity from the Seismocardiogram. Scientific Reports 2017;7(1):1-12.

[5] Inan OT, Migeotte PF, Park KS, Etemadi M, Tavakolian K, Casanella R, Zanetti J, Tank J, Funtova I, Prisk GK, Di Rienzo M. Ballistocardiography and Seismocardiography: A Review of Recent Advances. IEEE Journal of Biomedical and Health Informatics 2015;19(4):1414-1427.

[6] Kaisti M, Tadi MJ, Lahdenoja O, Hurnanen T, Saraste A, Pankaala M, Koivisto T. Stand-Alone Heartbeat Detection in Multidimensional Mechanocardiograms. IEEE Sensors Journal 2019;19(1):234-242.

[7] Hoog Antink C, Leonhardt S, Walter M. Reducing False Alarms in the ICU by Quantifying Self-Similarity of Multimodal Biosignals. Physiological Measurement 2016; 37(8):1233-1252.

[8] Hoog Antink C, Gao H, Brüser C, Leonhardt S. Beat-toBeat Heart Rate Estimation Fusing Multimodal Video and Sensor Data. Biomedical Optics Express 2015;6(8):28952907.

[9] Hoog Antink C, Leonhardt S, Walter M. Local Interval Estimation Improves Accuracy and Robustness of Heart Rate Variability Derivation from Photoplethysmography. In 41st Annual International Conference of the IEEE Engineering in Medicine and Biology Society. 2018; 3558-3561.

[10] Zink MD, Brüser C, Stüben BO, Napp A, Stöhr R, Leonhardt S, Marx N, Mischke K, Schulz JB, Schiefer J. Unobtrusive Nocturnal Heartbeat Monitoring by a Ballistocardiographic Sensor in Patients with Sleep Disordered Breathing. Scientific Reports 2017;7(1):1-13.

[11] Vollmer M, Bläsing D, Kaderali L. Alignment of MultiSensored Data: Adjustment of Sampling Frequencies and Time Shifts. Computing in Cardiology 2019;46.

Address for correspondence:

Christoph Hoog Antink

Medical Information Technology

Helmholtz-Institute, RWTH Aachen University

Pauwelsstr. 20 / D-52074 Aachen / Germany

hoog.antink@hia.rwth-aachen.de 